HAEMATOLOGICAL CANCER

\title{
Improvements with daratumumab
}

The standard regimens for patients with multiple myeloma (MM) include proteasome inhibitors (bortezomib or carfilzomib) and immunomodulatory agents. Daratumumab is a novel antibody that targets CD38, which is highly expressed in MM cells, and was approved by the FDA in 2015 as a monotherapy for patients with treatment-refractory MM after being shown to be safe and effective in this setting. "These findings urged us to combine daratumumab with standard therapy and investigate the efficacy of this combination in a prospective trial," explains Pieter Sonneveld, one of the investigators involved in a multicentre study with newly published results.

A total of 498 patients with relapsed and/or refractory MM received bortezomib and dexamethasone either supplemented with daratumumab (daratumumab group; $n=251$ ) or not (standard-ofcare group; $n=247$ ). The 12-month progression-free survival (PFS) rates were $60.7 \%$ and $26.9 \%$ in the daratumumab and standard-of-care groups, respectively. Median PFS was not reached in the daratumumab group versus 7.4 months in the standard-of-care group. The rates of overall response and very good partial response or better were both higher with daratumumab $(82.9 \%$ and $59.2 \%$, respectively), compared with the standard of care $(63.2 \%$ and 29.1\%).

Grade 3 or 4 adverse events were observed in $76.1 \%$ of patients in the daratumumab group and $62.4 \%$ in the standard-of-care group; these events included haematological adverse events, as well as peripheral sensory neuropathy. The treatment discontinuation rates owing to adverse events, however, were similar across both groups $\mathbf{( 7 . 4 \%}$ and $9.3 \%$, for the daratumumab versus the standard-of-care groups, respectively).

"The combination of daratumumab with bortezomib and dexamethasone will be practicechanging," Sonneveld opines. "Daratumumab will be tested with several other combinations in patients with both newly diagnosed and relapsed MM," he adds.

Diana Romero

ORIGINAL ARTICLE Palumbo, A. et al. Daratumumab, bortezomib and dexamethasone for multiple myeloma. N. Engl.J. Med. 375, 754-766 (2016)

FURTHER READING Usmani, S. Z. et al. Clinical

efficacy of daratumumab monotherapy in patients with heavily pretreated relapsed or refractory multiple myeloma. Blood 128, 37-44 (2016) 